\title{
EVALUTION OF THE PATIENS ORAL HEALTH RELATED QUALITY OF LIFE AFTER HARVESTING FREE GINGIVAL GRAFT
}

\section{ABSTRACT}

Objectives: Oral Health Related Quality of Life is the individual's perception of how oral health affects the quality of life and general health. Periodontal plastic surgery procedures have been reported to negatively affect the quality of life of patients after the operations. The aim of this study was to evaluate the effects of different treatment procedures applied for wound healing of the donor palate site after free gingival graft (FGG) operations on the quality of life.

Materials and Methods: After FGG harvesting, 60 patients' palatal donor sites were randomly assigned one of the six groups, giving 10 participants per group. Palatal wounds were treated with platelet-rich fibrin (PRF), essix retainer, ozone therapy, low-level laser therapy (LLLT) or collagen fleece. As a control group, palatal donor sites were left to secondary healing without any of the treatment procedures. After the postoperative procedures, The Oral Health Impact Profile (OHIP-14) questions were asked to patients to evaluate their quality of life, whereas parameters in relation to postoperative morbidity were analyzed by using Visual Analogue Scale (VAS).

Results: Regarding to the questions belonging Turkish version of OHIP-14 (OHIP14 TR), there were statistically significant relationships between the group categorical variables and the categorical results of question 7 and $10(p=0.002$ and $\mathrm{p}=0.015$ ). For these questions, the lowest scores were mostly given in the LLLT group. No statistically significant difference was observed between the study groups and total OHIP scores (TOHIP) and the means of 7 subscales of OHIP-14 ( $>0.05$ ). Significant differences were found between PRF-ozone groups on the 5 th day $(\mathrm{p}=0.011)$ for mean VAS scores exhibiting postoperative pain.

Conclusions: It has been observed that applying LLLT procedures to the donor wound area following FGG operations may have positive effects on the quality of life and PRF treatments may be more effective in terms of patient comfort after operation.

Key Words: Temostatic technics, laser therapy, oral surgical procedures, quality of life

\author{
(D)*Sıla Çağrı İşler ${ }^{1}$, \\ (iD) Ahu Uraz ${ }^{1}$, \\ (D)Janset Şengül ${ }^{1}$, \\ (i) Miray Çakıroğlu', \\ (D)Batuhan Bakırarar², \\ iD Deniz Çetiner ${ }^{1}$
}

ORCID IDs of the authors: S.Ç.I. $0000-0001-5419-9658$ A.U.0000-0001-6281-6855 J.Ş.0000-0001-5699-6781 M.C.0000-0002-6919-7986 B.B.0000-0002-5662-8193 D.Ç.0000-0002-1903-2999

${ }^{1}$ Department of Periodontology, Faculty of Dentistry, Gazi University, Ankara, Turkey. ${ }^{2}$ Department of Biostatistics, Faculty of Medicine, Ankara University, Ankara, Turkey.

$\begin{array}{ll}\text { Received } & : 13.08 .2018 \\ \text { Accepted } & : 13.12 .2018\end{array}$

\footnotetext{
How to Cite: İşler SÇ, Uraz A, Şengül J, Çakıroğlu M, Bakırarar B, Çetinder D. Evalutıon of the Patiens Oral Health Related Quality of Life After Harvesting Free Gingival Graft. Cumhuriyet Dent J 2019;22:1:11-21.

* Corresponding Author

Gazi University Faculty of Dentistry Department of Periodontology, 8. Cadde 82. Sokak Emek, 06510 Ankara Turkey,

Phone: +903122034243 Fax: +903122036020 E-mail: silaisler@gazi.edu.tr
} 


\section{INTRODUCTION}

Defined by the World Health Organization (WHO) as the individuals' perception of their positions in life with regard to their purposes, expectations, standards and concerns in the context of their respective culture and system of values, "quality of life" has gained importance by social studies since 1970s. ${ }^{1}$ While "health-related quality of life" is defined in connection with health and physical functions, emotional well-being, general perception of wellness and social functions ${ }^{2,3}$, Oral Health Related Quality of Life (OHRQoL) characterizes an individual's perception based on how their oral health affects their life standards and overall well-being. ${ }^{4-6}$ In other words, OHRQoL is a multidimensional concept that covers the variables that affect an individual's nutrition, sleep, communication with other individuals, their selfconfidence, and their satisfaction with their oral health. ${ }^{4}$ The factors that affect "quality of life" and OHRQoL include the psychological state, socialdemographic factors, life-style factors and the judgments of one's social circle. ${ }^{5}$

Until the last 30 years, physiological and psycho-social implications of oral health attracted less attention since they were not considered to have a major effect on the lives of individuals or cause a vital threat. However, it is a proven fact that oral health plays a major role in comfort, sleep, social life, aesthetic appearance, and consequently the quality of life. ${ }^{7}$ Even though periodontal diseases are not life-threatening conditions, they may not only affect eating, speaking and socializing but also interpersonal relationships, daily activities, hence "well-being" or "quality of life". ${ }^{8}$ Today, many studies have showed that just like other diseases, periodontal diseases have emotional and psycho-social implications. ${ }^{9-11}$ Oral Health Impact Profile-14 (OHIP-14) ${ }^{12}$ is a type of measurement that was designed to thoroughly identify the dysfunction, discomfort and injuries that are related to oral conditions. The questions in OHIP cover 7 dimensions, namely functional limitation, physical pain, psychological discomfort, physical disability, psychological disability, social disability and handicap, which are rooted and formulated in the theoretical model of oral health. ${ }^{12}$

An adequate width of attached gingiva is critical for maintaining periodontal/periimplant health. In case of a lack of adequate keratinized and attached gingiva, optimal plaque control cannot be achieved, and mucogingival stress in the relevant zone may cause gingival inflammation and recession. ${ }^{13}$

Several periodontal surgery techniques such as autogenous pedicle grafts, free gingival graft (FGG), connective tissue graft (CTG), epithelial connective tissue graft (ECTG) and guided tissue regeneration have been developed to increase the amount of keratinized attached gingiva and/or treat gingival recession. ${ }^{14}$

FGG is a predictable and successful technique in treating mucogingival problems such as insufficient attached gingiva and also gingival recession. While the palatal zone is usually chosen as the donor site, the palatal donor site heals by secondary intention after FGG operations, and the healing process may take a few weeks. ${ }^{15}$ Postoperative complaints continue until the epithelization is completed within 2 to 4 weeks. ${ }^{16}$ The most frequent complications after FGG applications are hemorrhage, pain, burning sensation, sensitivity, paresthesia, mucocele, herpetic lesion in the palate, and the delay in wound healing. ${ }^{17,18}$ Such problems may restrict patients' daily activities such as eating, drinking, brushing teeth or speaking, and affect their quality of life. A lot of methods including the implementation of hemostatic agents, bioactive materials, antibacterial and antiseptic agents, herbal products, platelet-rich fibrin (PRF) and low-level laser treatment (LLLT) have been used to minimize the discomfort and accelerate wound healing in this recovery process. ${ }^{19-23}$ Therefore, the purpose of this study was to assess the effects of different treatment procedures applied for wound healing of the donor palate site after the FGG operation on the oral health and quality of life. 


\section{MATERIALS AND METHODS}

\section{Selection of Patients and Study Protocols}

The research protocol was approved by the Clinical Research Ethics Committee of Ankara University, Faculty of Dentistry (36290600/114). A total of 60 patients aged between 18 and 65, who admitted to Gazi University, Faculty of Dentistry, had shallow vestibule along with the inadequate width of attached gingiva and were indicated with FGG procedure for isolated gingival recession defects on mandibular and maxillary anterior teeth, were included in the present study. Informed written consent forms were obtained for all participants before their participation.

The patients who were included in the study were non-smokers, did not have any systemic condition that may affect wound healing, did have a full mouth plaque scores (FMPS) and full mouth bleeding scores (FMBS) of less than $20 \%$, did not undergo any periodontal treatment in the previous 6 months, did not use any antibiotics in the previous 3 months, were not in the lactation period, and did not undergo any surgical intervention for their operation area. The patients who failed to fulfill these criteria were excluded from the study.

Before the surgery, all patients received oral hygiene instructions and the Phase I periodontal treatment including scaling and root planning and polishing of the teeth. All patients were recommended to use a standardized soft toothbrush according to a standardized method of brushing (the modified Stillman technique).

Four weeks after the initial therapy, FGG procedures were performed by the same periodontist as described by Sullivan and Atkins ${ }^{24}$ and modified by Miller ${ }^{25}$. To prepare the donor area, a rectangular-shape incision with $1-1.5 \mathrm{~mm}$ thickness was made, and care was taken to place most of the coronal part of the incision at least 2 $\mathrm{mm}$ apical from gingival margins of the upper teeth. The apical coronal dimension of the graft was standardized (9x11 mm) using a sterile aluminum foil template due to the selection of the recessions on the mandibular and maxillary anterior teeth. After removing the FGG, two vertical mattress sutures were made mesial and distal to the tissue-harvesting site to prevent immediate and/or delayed bleeding using 3/0 resorbable sutures (Pegesorb, Doğsan, Istanbul, Turkey).

60 patients were randomly allocated into one of the six study groups, using a computer-generated randomization table, based on the applications of treatment methods on the palatal donor site following FGG procedures.

a) Group 1 (Control) $(\mathrm{n}=10)$ : The palatal donor site was left spontaneously for secondary healing.

b) Group 2 (PRF) $(\mathrm{n}=10)$ : PRF membranes prepared as described in the method developed by Choukroun ${ }^{26}$ were applied to the palatal donor site by vertical mattress sutures using 3/0 resorbed sutures (Pegesorb, Doğsan, İstanbul, Turkey).

c) Group 3 (Essix retainer) $(\mathrm{n}=10)$ : 1-mm-thick Essix retainer (Clear Advantages Series, Ortho Technology, Florida, United States) prepared preoperatively to cover the palatal donor site was used.

d) Group 4 (Ozone) $(\mathrm{n}=10)$ : Topical gaseous ozone (Ozone DTA Ozone Generator, DentaTec Dental AS, Hov, Norway) with an oxygen power of $80 \%$ and a concentration of $2100 \mathrm{ppm}$ was administered to the palatal donor site for 30 seconds on the day 1,3 , and 7 after the operation.

e) Group 5 (LLLT) $(n=10)$ : LLLT was applied to the palatal donor site for 30 seconds using a diode laser $(\lambda=970 \pm 15 \mathrm{~nm})$ (SIROLaser Xtend, Sirona Dental Systems GmbH, Bensheim, Germany) (tissue dose: $5.25 \mathrm{~J} / \mathrm{cm}^{2}$ and power: $2 \mathrm{~W}$ ) on the day 1,3 , and 7 after the operation.

f) Group 6 (Collagen fleece) $(n=10)$ : The palatal donor site was covered with a collagen sponge (Bego Collagen Fleece, Bremen, Germany) and immobilized using a 3/0 resorbed suture (Pegasorb) and a vertical mattress suture.

Postoperative care was aimed at maintaining wound stability. In order to control for postoperative infections, the patients were asked to use a $0.12 \%$ chlorhexidine mouth rinse (Kloroben, Drogsan, Istanbul, Turkey) twice a day for three weeks, postoperatively. Patients were prescribed $100 \mathrm{mg}$ flurbiprofen tablets (Majezik, Sanovel, 
Istanbul, Turkey) to take systemically up to three times/day for a week, in case of pain.

\section{Clinical Evaluations}

The Plaque index (PI), ${ }^{27}$ Gingival index $(\mathrm{GI}),{ }^{28}$ Bleeding on probing (BOP) and Probing depth (PD) measurements were taken pre-operatively to identify the periodontal health of patients. The measurements were obtained from four aspects of the teeth-mesial, distal, buccal, and lingual/palatinal surfaces-using a Williams periodontal probe (Hu-Friedy, Chicago, IL, Amerika) by the same study examiner.

Furthermore, the periodontal probe was used to measure the distance from the gingival margin to the cemento-enamel junction (CEJ) which was identified as the Recession depth (RD) and Recession width (RW) was measured as the distance from one border of the recession to another in mesiodistal direction at $1 \mathrm{~mm}$ apical of the CEJ. Palatal tissue thickness (PTT) and the size and thickness of the grafts were also recorded during the surgical procedure.

Postoperative instructions included to discontinue toothbrushing and flossing around the surgical sites during the first 14 days after surgery. During this period, patients were instructed to rinse with a $0.12 \%$ chlorhexidine gluconate solution (Kloroben, Drogsan, Ankara, Turkey) two times a day. Patients were also prescribed $100 \mathrm{mg}$ flurbiprofen tablets (Majezik, Sanovel, Istanbul, Turkey) to take systemically up to three times/day for a week, in case of pain.

OHRQoL was evaluated using the Turkish version of OHIP-14 (OHIP-14 TR) scale ${ }^{29}$ which consists of 14 items and 7 sub-scales (1) functional limitation, 2) physical pain, 3) psychological discomfort, 4) physical disability, 5) psychological disability, 6) social disability and 7) handicap. The Likert-type scale ( $0=$ "Never", $1=$ "Rarely", $2=$ "Sometimes", 3="Often", 4="Very Often") was used. OHIP-14 TR scale was implemented face-toface by the same researcher on the 14th day postoperatively.

Post-operative pain was also assessed using a questionnaire showing the intensity of the given Table 1. Patients' Demographic Data and Clinical Periodontal Parameters event on a visual analogic scale (VAS). VAS is a scale by which the severity of the measured values is rated on a $10-\mathrm{cm}$ scale (0: no pain, 1: minimal pain, 10: severe pain). This assessment was made every day for the first 7 days and on the 14th day following the FGG operation.

\section{Statistical Analysis}

The data was analyzed using the statistics packages SPSS 20 (IBM Corp. Released 2011. IBM SPSS Statistics for Windows, Version 20.0. Armonk, NY: IBM Corp.) and R (Version 3.0.2). As descriptive statistics mean \pm standard deviation and median (minimum-maximum) was used for quantitative variables, and numbers (percentage) were used for qualitative variables. For quantitative variables, whether there was a statistically significant difference among the categories of qualitative variables which have more than two categories was decided using One-Way Analysis of Variance (ANOVA) where normal distribution assumptions were established, and Kruskal Wallis $\mathrm{H}$ test where it they were not established. Chi-square test was used to examine the relation between the two qualitative variables. The level of statistical significance was 0.05 .

\section{RESULTS}

60 patients aged 18 to $65(39.5 \pm 12.7)$ were included in our study. 44 of them $(73.3 \%)$ were female, and 16 (26.7\%) were male. All patients regularly attended the examinations, and none of them left the study. No postoperative complications were reported by the patients in terms of delayed bleeding. The presence of partial necrosis was observed in one patient from control group and one patient from collagen fleece group.

The demographic data and clinical periodontal parameters of the patients are shown in Table 1. No statistically significant difference was found in terms of age and gender of the patients in comparison among the groups $(p=0.993$ and $p=0.325$ ). No statistically significant difference was seen in the measurements of PI, GI, PD, BOP, $\mathrm{RD}, \mathrm{RW}$, and PTT with regard to clinical parameters $(p>0.05)$ (Table 1). 
İşler SÇ. et al.

\begin{tabular}{|c|c|c|c|c|c|c|c|c|}
\hline \multicolumn{2}{|c|}{ Parameters } & $\begin{array}{l}\text { Group } 1 \\
\text { Control }\end{array}$ & $\begin{array}{l}\text { Group } 2 \\
\text { PRF }\end{array}$ & $\begin{array}{l}\text { Group } 3 \\
\text { Essix } \\
\text { retainer }\end{array}$ & $\begin{array}{l}\text { Group } 4 \\
\text { Ozone }\end{array}$ & $\begin{array}{l}\text { Group } 5 \\
\text { LLLT }\end{array}$ & $\begin{array}{l}\text { Group } 6 \\
\text { Collagen } \\
\text { fleece }\end{array}$ & $P$ value \\
\hline \multirow[b]{2}{*}{ Age } & Mean \pm SD & $40.0 \pm 15.7$ & $40.4 \pm 16.0$ & $39.6 \pm 6.2$ & $37.0 \pm 11.3$ & $40.3 \pm 11.3$ & $39.7 \pm 15.8$ & \multirow[b]{2}{*}{$0.993^{*}$} \\
\hline & $\begin{array}{c}\text { Median } \\
\text { (Min.-Max.) }\end{array}$ & $\begin{array}{c}33.0 \\
(18.0-65.0)\end{array}$ & $\begin{array}{c}44.0 \\
(18.0-63.0)\end{array}$ & $\begin{array}{c}37.5 \\
(31.0-51.0)\end{array}$ & $\begin{array}{c}37.5 \\
(31.0-51.0)\end{array}$ & $\begin{array}{c}39.0 \\
(21.0-56.0)\end{array}$ & $\begin{array}{c}42.0 \\
(22.0-65.0)\end{array}$ & \\
\hline \multirow{2}{*}{ Gender } & Female & $9(90.0)$ & $6(60.0)$ & $9(90.0)$ & $7(70.0)$ & $5(50.0)$ & $8(80.0)$ & \multirow{2}{*}{$0.325^{* *}$} \\
\hline & Male & $1(10.0)$ & $4(40.0)$ & $1(10.0)$ & $3(30.0)$ & $5(50.0)$ & $2(20.0)$ & \\
\hline \multirow[b]{2}{*}{ PI } & Mean \pm SD & $10.5 \pm 4.5$ & $9.5 \pm 6.4$ & $9.0 \pm 5.2$ & $9.6 \pm 4.6$ & $8.2 \pm 6.3$ & $7.3 \pm 6.9$ & \multirow[b]{2}{*}{$0.494^{\#}$} \\
\hline & $\begin{array}{c}\text { Median } \\
\text { (Min.-Max.) }\end{array}$ & $\begin{array}{c}10.3 \\
(4.8-21.3)\end{array}$ & $\begin{array}{c}8.1 \\
(2.1-21.3)\end{array}$ & $\begin{array}{c}7.7 \\
(3.6-20.0)\end{array}$ & $\begin{array}{c}9.8 \\
(3.6-19.2)\end{array}$ & $\begin{array}{c}7.3 \\
(0.0-18.2)\end{array}$ & $\begin{array}{c}5.5 \\
(0.0-25.0)\end{array}$ & \\
\hline \multirow[b]{2}{*}{ GI } & Mean \pm SD & $0.2 \pm 0.2$ & $0.2 \pm 0.1$ & $0.2 \pm 0.2$ & $0.1 \pm 0.1$ & $0.1 \pm 0.1$ & $0.1 \pm 0.1$ & \multirow[b]{2}{*}{$0.541^{\#}$} \\
\hline & $\begin{array}{c}\text { Median } \\
\text { (Min.-Max.) }\end{array}$ & $\begin{array}{c}0.2 \\
(0.0-0.5) \\
\end{array}$ & $\begin{array}{c}0.1 \\
(0.1-0.3) \\
\end{array}$ & $\begin{array}{c}0.1 \\
(0.0-0.6) \\
\end{array}$ & $\begin{array}{c}0.1 \\
(0.0-0.3) \\
\end{array}$ & $\begin{array}{c}0.1 \\
(0.0-0.3) \\
\end{array}$ & $\begin{array}{c}0.1 \\
(0.0-0.2) \\
\end{array}$ & \\
\hline \multirow[b]{2}{*}{ PD mm } & Mean \pm SD & $1.9 \pm 0.7$ & $1.9 \pm 0.2$ & $1.9 \pm 0.2$ & $1.9 \pm 0.2$ & $1.8 \pm 0.4$ & $1.7 \pm 0.6$ & \multirow{2}{*}{$0.223^{\#}$} \\
\hline & $\begin{array}{c}\text { Median } \\
\text { (Min.-Max.) }\end{array}$ & $\begin{array}{c}2.1 \\
(0.0-2.6) \\
\end{array}$ & $\begin{array}{c}1.9 \\
(1.6-2.2) \\
\end{array}$ & $\begin{array}{c}1.9 \\
(1.6-2.1) \\
\end{array}$ & $\begin{array}{c}1.9 \\
(1.6-2.4) \\
\end{array}$ & $\begin{array}{c}1.8 \\
(1.5-2.9) \\
\end{array}$ & $\begin{array}{c}1.8 \\
(0.0-2.4) \\
\end{array}$ & \\
\hline \multirow[b]{2}{*}{ BOP \% } & Mean \pm SD & $11.1 \pm 5.2$ & $9.3 \pm 4.3$ & $7.6 \pm 5.0$ & $9.8 \pm 5.5$ & $9.0 \pm 6.6$ & $8.2 \pm 5.6$ & \multirow[b]{2}{*}{$0.394^{\#}$} \\
\hline & $\begin{array}{c}\text { Median } \\
\text { (Min.-Max.) }\end{array}$ & $\begin{array}{c}11.5 \\
(1.7-22.2) \\
\end{array}$ & $\begin{array}{c}9.1 \\
(4.3-17.6) \\
\end{array}$ & $\begin{array}{c}7.5 \\
(2.0-19.4) \\
\end{array}$ & $\begin{array}{c}8.5 \\
(4.5-22.8) \\
\end{array}$ & $\begin{array}{c}7.4 \\
(2.9-25.9) \\
\end{array}$ & $\begin{array}{c}7.7 \\
(1.9-22.2) \\
\end{array}$ & \\
\hline \multirow[b]{2}{*}{$\mathrm{RD} \mathrm{mm}$} & Mean \pm SD & $4.0 \pm 1.8$ & $3.9 \pm 1.6$ & $4.2 \pm 1.8$ & $4.5 \pm 1.9$ & $4.8 \pm 1.9$ & $2.6 \pm 1.3$ & \multirow{2}{*}{$0.120^{\#}$} \\
\hline & $\begin{array}{c}\text { Median } \\
\text { (Min.-Max.) }\end{array}$ & $\begin{array}{c}4.0 \\
(1.5-7.0) \\
\end{array}$ & $\begin{array}{c}3.5 \\
(1.0-7.0) \\
\end{array}$ & $\begin{array}{c}3.8 \\
(2.0-7.0) \\
\end{array}$ & $\begin{array}{c}5.0 \\
(1.0-8.0) \\
\end{array}$ & $\begin{array}{c}5.0 \\
(2.0-9.0) \\
\end{array}$ & $\begin{array}{c}3.0 \\
(0.0-4.0) \\
\end{array}$ & \\
\hline \multirow[b]{2}{*}{$\mathrm{RW} \mathrm{mm}$} & Mean \pm SD & $3.1 \pm 0.9$ & $3.1 \pm 0.7$ & $3.1 \pm 1.4$ & $3.9 \pm 1.4$ & $3.2 \pm 0.9$ & $2.3 \pm 1.2$ & \multirow[b]{2}{*}{$0.097^{\#}$} \\
\hline & $\begin{array}{c}\text { Median } \\
\text { (Min.-Max.) }\end{array}$ & $\begin{array}{c}3.0 \\
(2.0-5.0) \\
\end{array}$ & $\begin{array}{c}3,0 \\
(2.0-4.0) \\
\end{array}$ & $\begin{array}{c}3.0 \\
(2.0-7.0) \\
\end{array}$ & $\begin{array}{c}3.0 \\
(3.0-7.0) \\
\end{array}$ & $\begin{array}{c}3.0 \\
(2.0-5.0) \\
\end{array}$ & $\begin{array}{c}3.0 \\
(0.0-3.0) \\
\end{array}$ & \\
\hline \multirow[b]{2}{*}{ PTT mm } & Mean \pm SD & $3.9 \pm 0.6$ & $3.9 \pm 0.7$ & $3.4 \pm 0.4$ & $3.2 \pm 0.7$ & $3.4 \pm 0.3$ & $3.9 \pm 0.9$ & \multirow[b]{2}{*}{$0.051^{\#}$} \\
\hline & $\begin{array}{c}\text { Median } \\
\text { (Min.-Max.) }\end{array}$ & $\begin{array}{c}4.2 \\
(3.0-5.0) \\
\end{array}$ & $\begin{array}{c}4.0 \\
(2.5-5.0) \\
\end{array}$ & $\begin{array}{c}3.3 \\
(3.0-4.0) \\
\end{array}$ & $\begin{array}{c}3.0 \\
(2.0-4.0) \\
\end{array}$ & $\begin{array}{c}3.2 \\
(3.1-4.1) \\
\end{array}$ & $\begin{array}{c}3.5 \\
(3.0-5.5) \\
\end{array}$ & \\
\hline
\end{tabular}

*: One-way ANOVA, \#:Kruskal Wallis H test, **: Chi-square test. SD: Standard deviation; PRF: Platelet-rich fibrin; LLLT: Low-level laser therapy; PI: Plaque index; GI: Gingival index; PD: Probing depth; BOP: Bleeding on probing; RD: Recession depth; RW: Recession width; PTT: Palatal tissue thickness.

Table 2. Intergroup analysis of OHIP-14 TR questionnaires

\begin{tabular}{|c|c|c|c|c|c|c|c|c|c|c|c|c|c|c|c|c|c|c|c|c|c|c|c|c|c|c|c|c|c|c|c|}
\hline OHIP-14 & \multicolumn{5}{|c|}{ Group I Control } & \multicolumn{5}{|c|}{ Group 2 PRF } & \multicolumn{5}{|c|}{ Group 3 Essix retainer } & \multicolumn{5}{|c|}{ Group 4 Ozone } & \multicolumn{5}{|c|}{ Group 5 LLLT } & \multicolumn{5}{|c|}{ Group 6 Collagen fleece } & $P$ \\
\hline \multirow[t]{2}{*}{ Items / Questions } & \multicolumn{5}{|l|}{$\%$} & \multicolumn{5}{|l|}{$9 \%$} & \multicolumn{5}{|l|}{$\%$} & \multicolumn{5}{|l|}{$\begin{array}{l}\% \\
\end{array}$} & \multicolumn{5}{|l|}{$9 \%$} & \multicolumn{5}{|l|}{$\%$} & \multirow[t]{2}{*}{ 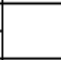 } \\
\hline & $\begin{array}{ll}0 \\
\end{array}$ & 1 & \begin{tabular}{|l|}
2 \\
\end{tabular} & 3 & 4 & 0 & 1 & 2 & 3 & 4 & 0 & 1 & 2 & 3 & 4 & 0 & 1 & 2 & 3 & 4 & 0 & 1 & 2 & 3 & 4 & 0 & 1 & 2 & 3 & 4 & \\
\hline $\begin{array}{l}\text { OH-1/Trouble } \\
\text { pronouncing } \\
\text { words }\end{array}$ & 60 & 30 & 10 & 0 & 0 & 40 & 30 & 10 & 10 & 10 & 30 & 30 & 30 & 10 & 0 & 20 & 30 & 20 & 20 & 10 & 30 & 20 & 40 & 10 & 0 & 20 & 30 & 30 & 20 & 0 & 0.916 \\
\hline $\begin{array}{l}\text { OH-2 / Sense of } \\
\text { taste worse }\end{array}$ & 40 & 20 & 40 & 0 & 0 & 50 & 20 & 20 & 0 & 10 & 20 & 50 & 30 & 0 & 0 & 30 & 10 & 40 & 10 & 10 & 40 & 20 & 30 & 0 & 10 & 40 & 10 & 20 & 30 & 0 & 0.637 \\
\hline $\begin{array}{l}\text { OH-3 / Painful } \\
\text { aching in the } \\
\text { mouth? }\end{array}$ & 50 & 30 & 20 & 0 & 0 & 50 & 30 & 20 & 0 & 0 & 60 & 30 & 10 & 0 & 0 & 20 & 40 & 40 & 0 & 0 & 60 & 20 & 20 & 0 & 0 & 60 & 0 & 30 & 10 & 0 & 0.511 \\
\hline $\begin{array}{l}\mathrm{OH}-4 / \\
\text { Uncomfortable } \\
\text { to eat }\end{array}$ & 40 & 40 & 20 & 0 & 0 & 60 & 30 & 10 & 0 & 0 & 50 & 10 & 30 & 10 & 0 & 40 & 30 & 20 & 0 & 10 & 80 & 20 & 20 & 0 & 0 & 50 & 0 & 20 & 20 & 10 & 0.362 \\
\hline $\begin{array}{l}\text { OH-5/ Self- } \\
\text { conscious }\end{array}$ & 40 & 40 & 20 & 0 & 0 & 40 & 40 & 20 & 0 & 0 & 60 & 10 & 20 & 0 & 10 & 50 & 10 & 30 & 10 & 0 & 60 & 30 & 0 & 10 & 0 & 70 & 10 & 10 & 10 & 0 & 0.774 \\
\hline OH-6 / Felt tense & 40 & 40 & 20 & 0 & 0 & 80 & 10 & 10 & 0 & 0 & 40 & 0 & 40 & 10 & 10 & 50 & 0 & 40 & 0 & 10 & 70 & 20 & 10 & 0 & 0 & 60 & 0 & 20 & 10 & 10 & 0.200 \\
\hline $\begin{array}{l}\text { OH-7/ } \\
\text { Unsatisfactory } \\
\text { diet }\end{array}$ & 40 & 30 & 30 & 0 & 0 & 20 & 60 & 20 & 0 & 0 & 20 & 60 & 20 & 0 & 0 & 50 & 10 & 20 & 20 & 0 & 80 & 0 & 20 & 0 & 0 & 20 & 0 & 40 & 40 & 0 & $0.002^{\circ}$ \\
\hline $\begin{array}{l}\text { OH-8 / Had to } \\
\text { interrupt meals }\end{array}$ & 40 & 40 & 10 & 10 & 0 & 60 & 20 & 20 & 0 & 0 & 50 & 10 & 40 & 0 & 0 & 50 & 10 & 30 & 0 & 10 & 20 & 40 & 40 & 0 & 0 & 40 & 30 & 10 & 20 & 0 & 0.446 \\
\hline $\begin{array}{l}\text { OH-9/ Difficult } \\
\text { to relax }\end{array}$ & 70 & 20 & 10 & 0 & 0 & 70 & 20 & 0 & 10 & 0 & 70 & 10 & 10 & 0 & 10 & 70 & 10 & 10 & 0 & 10 & 50 & 20 & 30 & 0 & 0 & 50 & 10 & 30 & 0 & 10 & 0.590 \\
\hline $\begin{array}{l}\text { OH-10 / } \\
\text { Embarrassed }\end{array}$ & 40 & 40 & 20 & 0 & 0 & 90 & 0 & 10 & 0 & 0 & 90 & 10 & 0 & 0 & 0 & 90 & 10 & 0 & 0 & 0 & 100 & 0 & 0 & 0 & 0 & 60 & 10 & 30 & 0 & 0 & $0.015^{4}$ \\
\hline $\begin{array}{l}\text { OH-1l/ } \\
\text { Irritability with } \\
\text { other people }\end{array}$ & 60 & 20 & 20 & 0 & 0 & 70 & 30 & 0 & 0 & 0 & 90 & 10 & 0 & 0 & 0 & 90 & 0 & 0 & 10 & 0 & 80 & 0 & 20 & 0 & 0 & 80 & 0 & 20 & 0 & 0 & 0.229 \\
\hline $\begin{array}{l}\text { OH-12/ } \\
\text { Difficulty doing } \\
\text { your usual jobs }\end{array}$ & 70 & 20 & 10 & 0 & 0 & 60 & 40 & 0 & 0 & 0 & 80 & 10 & 0 & 10 & 0 & 80 & 10 & 0 & 10 & 0 & 90 & 0 & 10 & 0 & 0 & 50 & 10 & 10 & 30 & 0 & 0.225 \\
\hline $\begin{array}{l}\text { OH-13 / Felt life } \\
\text { less satisfying }\end{array}$ & 60 & 30 & 10 & 0 & 0 & 100 & 0 & 0 & 0 & 0 & 90 & 10 & 0 & 0 & 0 & 90 & 10 & 0 & 0 & 0 & 70 & 30 & 0 & 0 & 0 & 70 & 10 & 10 & 10 & 0 & 0.349 \\
\hline $\begin{array}{l}\text { OH-14 / Totally } \\
\text { unable to } \\
\text { function }\end{array}$ & 60 & 30 & 10 & 0 & 0 & 90 & 10 & 0 & 0 & 0 & 80 & 20 & 0 & 0 & 0 & 80 & 10 & 10 & 0 & 0 & 80 & 0 & 20 & 0 & 0 & 80 & 0 & 20 & 0 & 0 & 0.681 \\
\hline
\end{tabular}

*: p<0.05, Chi-square test. PRF: Platelet-rich fibrin; LLLT: Low-level laser therapy; OHIP-14: Oral Health Impact Profile-14. 
A statistically significant relation was found between the qualitative variables of the group and the qualitative variables of the question 7 and question 10 related to the OHIP-14 TR scale $(p=0.002$ and $p=0.015)$. For all groups, no patient chose " 4 " for the question 7. The highest number of patients who chose the rating "3" was in the group which was administered collagen fleece (40\%). The highest number of patients who chose the rating "0", which was the lowest rating, was in the LLLT group (80\%) for the question 7. All patients in the LLLT group chose "0" for the question 10 (Table 2).

The 14th-day the mean of Total OHIP (TOHIP) scores varied between $6.70 \pm 4.4$ and $14.80 \pm 9.0$ for the study groups (Table 3).

Table 3. Intergroup evaluations of the mean of the seven major subscales of the OHIP-14 TR scale

\begin{tabular}{|c|c|c|c|c|c|}
\hline OHIP-14 & Groups & $\mathbf{N}$ & Mean \pm SD & Median (Min.Max.) & $P$ value \\
\hline \multirow{6}{*}{$\begin{array}{l}\text { Functional } \\
\text { limitation }\end{array}$} & Control & 10 & $1.5 \pm 1.1$ & $1.5(0.0-3.0)$ & \multirow{6}{*}{0.538} \\
\hline & PRF & 10 & $2.2 \pm 2.3$ & $1.0(0.0-6.0)$ & \\
\hline & Essix retainer & 10 & $2.3 \pm 1.4$ & $2.0(0.0-5.0)$ & \\
\hline & Ozone & 10 & $3.3 \pm 2.5$ & $3.0(0.0-8.0)$ & \\
\hline & LLLT & 10 & $2.5 \pm 2.2$ & $2.0(0.0-6.0)$ & \\
\hline & Collagen fleece & 10 & $2.9 \pm 2.0$ & $3.0(0.0-5.0)$ & \\
\hline \multirow{6}{*}{ Physical pain } & Control & 10 & $1.5 \pm 1.4$ & $1.0(0.0-3.0)$ & \multirow{6}{*}{0.376} \\
\hline & PRF & 10 & $1.2 \pm 1.1$ & $1.0(0.0-3.0)$ & \\
\hline & Essix retainer & 10 & $1.5 \pm 1.6$ & $1.0(0.0-4.0)$ & \\
\hline & Ozone & 10 & $2.3 \pm 1.7$ & $2.0(0.0-6.0)$ & \\
\hline & LLLT & 10 & $0.8 \pm 1.0$ & $0.5(0.0-3.0)$ & \\
\hline & Collagen fleece & 10 & $2.3 \pm 2.5$ & $2.0(0.0-7.0)$ & \\
\hline \multirow{6}{*}{$\begin{array}{l}\text { Psychological } \\
\text { discomfort }\end{array}$} & Control & 10 & $1.6 \pm 1.3$ & $1.5(0.0-4.0)$ & \multirow{6}{*}{0.507} \\
\hline & PRF & 10 & $1.1 \pm 2.0$ & $0.0(0.0-6.0)$ & \\
\hline & Essix retainer & 10 & $2.4 \pm 2.6$ & $2.5(0.0-8.0)$ & \\
\hline & Ozone & 10 & $2.2 \pm 2.0$ & $2.0(0.0-6.0)$ & \\
\hline & LLLT & 10 & $1.0 \pm 1.3$ & $0.5(0.0-4.0)$ & \\
\hline & Collagen fleece & 10 & $1.7 \pm 2.5$ & $0.0(0.0-6.0)$ & \\
\hline \multirow{6}{*}{ Physical disability } & Control & 10 & $1.8 \pm 1.7$ & $1.5(0.0-5.0)$ & \multirow{6}{*}{0.540} \\
\hline & PRF & 10 & $1.6 \pm 1.3$ & $1.5(0.0-4.0)$ & \\
\hline & Essix retainer & 10 & $1.9 \pm 0.9$ & $2.0(1.0-3.0)$ & \\
\hline & Ozone & 10 & $2.2 \pm 2.6$ & $1.0(0.0-7.0)$ & \\
\hline & LLLT & 10 & $1.6 \pm 1.4$ & $1.0(0.0-4.0)$ & \\
\hline & Collagen fleece & 10 & $3.1 \pm 2.1$ & $3.0(0.0-6.0)$ & \\
\hline \multirow{6}{*}{$\begin{array}{l}\text { Functional } \\
\text { limitation }\end{array}$} & Control & 10 & $1.5 \pm 1.1$ & $1.5(0.0-3.0)$ & \multirow{6}{*}{0.538} \\
\hline & PRF & 10 & $2.2 \pm 2.3$ & $1.0(0.0-6.0)$ & \\
\hline & Essix retainer & 10 & $2.3 \pm 1.4$ & $2.0(0.0-5.0)$ & \\
\hline & Ozone & 10 & $3.3 \pm 2.5$ & $3.0(0.0-8.0)$ & \\
\hline & LLLT & 10 & $2.5 \pm 2.2$ & $2.0(0.0-6.0)$ & \\
\hline & Collagen fleece & 10 & $2.9 \pm 2.0$ & $3.0(0.0-5.0)$ & \\
\hline \multirow{6}{*}{ Physical pain } & Control & 10 & $1.5 \pm 1.4$ & $1.0(0.0-3.0)$ & \multirow{6}{*}{0.376} \\
\hline & PRF & 10 & $1.2 \pm 1.1$ & $1.0(0.0-3.0)$ & \\
\hline & Essix retainer & 10 & $1.5 \pm 1.6$ & $1.0(0.0-4.0)$ & \\
\hline & Ozone & 10 & $2.3 \pm 1.7$ & $2.0(0.0-6.0)$ & \\
\hline & LLLT & 10 & $0.8 \pm 1.0$ & $0.5(0.0-3.0)$ & \\
\hline & Collagen fleece & 10 & $2.3 \pm 2.5$ & $2.0(0.0-7.0)$ & \\
\hline \multirow{6}{*}{$\begin{array}{l}\text { Psychological } \\
\text { discomfort }\end{array}$} & Control & 10 & $1.6 \pm 1.3$ & $1.5(0.0-4.0)$ & \multirow{6}{*}{0.507} \\
\hline & PRF & 10 & $1.1 \pm 2.0$ & $0.0(0.0-6.0)$ & \\
\hline & Essix retainer & 10 & $2.4 \pm 2.6$ & $2.5(0.0-8.0)$ & \\
\hline & Ozone & 10 & $2.2 \pm 2.0$ & $2.0(0.0-6.0)$ & \\
\hline & LLLT & 10 & $1.0 \pm 1.3$ & $0.5(0.0-4.0)$ & \\
\hline & Collagen fleece & 10 & $1.7 \pm 2.5$ & $0.0(0.0-6.0)$ & \\
\hline \multirow{6}{*}{ Physical disability } & Control & 10 & $1.8 \pm 1.7$ & $1.5(0.0-5.0)$ & \multirow{6}{*}{0.540} \\
\hline & PRF & 10 & $1.6 \pm 1.3$ & $1.5(0.0-4.0)$ & \\
\hline & Essix retainer & 10 & $1.9 \pm 0.9$ & $2.0(1.0-3.0)$ & \\
\hline & Ozone & 10 & $2.2 \pm 2.6$ & $1.0(0.0-7.0)$ & \\
\hline & LLLT & 10 & $1.6 \pm 1.4$ & $1.0(0.0-4.0)$ & \\
\hline & Collagen fleece & 10 & $3.1 \pm 2.1$ & $3.0(0.0-6.0)$ & \\
\hline
\end{tabular}

SD: Standard deviation; PRF: Platelet-rich fibrin; LLLT: Low-level laser therapy; OHIP-14: Oral Health Impact Profile-14; TOHIP: Total OHIP scores 
Seven sub-scale values of the OHIP-14 questionnaire did not show any statistically significant difference among the groups $(p>0.05)$. It was found that the mean functional limitation score was highest (3.3 \pm 2.5$)$ in the ozone group and lowest $(1.5 \pm 1.1)$ in the control group. The mean physical pain score was highest $(2.3 \pm 2.5)$ in the collagen fleece group and lowest $(0.8 \pm 1.0)$ in the LLLT group. The mean psychological discomfort score was highest in the essix group (2.4 \pm 2.6$)$ and lowest in the LLLT group (1.0 1.3$)$. While in terms of physical and psychological disability had higher values in the collagen fleece group compared to the other groups, the mean physical disability scores were lowest in the PRF and LLLT groups, and the mean psychological disability score was lowest in the essix retainer group.
While handicap disturbed the patients in the collagen fleece group the most, it disturbed the patients in the PRF group the least.

The statistically significant difference was observed between PRF and ozone groups for the mean VAS values regarding postoperative pain only on day $5(p=0.011)$. Although there was a tendency towards a lower VAS value in the PRF group compared to other groups for all study follow-up periods, all inter-group comparisons did not show any statistical significance on the other postoperative days $(p>0.05)$ (Table 4). At the 14day follow-up, the patients for all the groups reported no postoperative pain.

Table 4. Comparison of the mean VAS values regarding postoperative pain between the groups

\begin{tabular}{|c|c|c|c|c|c|}
\hline VAS & Groups & $\mathbf{N}$ & $\operatorname{Mean} \pm$ SD & Median (Min.-Max.) & $P$ values \\
\hline \multirow{6}{*}{ Day 1} & Control & 10 & $2.2 \pm 3.4$ & $0.0(0.0-10.0)$ & \multirow{6}{*}{0.578} \\
\hline & PRF & 10 & $0.7 \pm 1.5$ & $0.0(0.0-4.0)$ & \\
\hline & Essix retainer & 10 & $0.9 \pm 2.0$ & $0.0(0.0-6.0)$ & \\
\hline & Ozone & 10 & $0.9 \pm 1.7$ & $0.0(0.0-5.0)$ & \\
\hline & LLLT & 10 & $1.9 \pm 2.7$ & $1.5(0.0-9.0)$ & \\
\hline & Collagen fleece & 10 & $1.3 \pm 2.7$ & $0.0(0.0-8.0)$ & \\
\hline \multirow{6}{*}{ Day 2} & Control & 10 & $2.1 \pm 3.0$ & $1.0(0.0-10.0)$ & \multirow{6}{*}{0.378} \\
\hline & PRF & 10 & $0.3 \pm 0.5$ & $0.0(0.0-1.0)$ & \\
\hline & Essix retainer & 10 & $0.6 \pm 1.0$ & $0.0(0.0-3.0)$ & \\
\hline & Ozone & 10 & $1.3 \pm 1.9$ & $0.0(0.0-5.0)$ & \\
\hline & LLLT & 10 & $0.9 \pm 1.4$ & $0.0(0.0-4.0)$ & \\
\hline & Collagen fleece & 10 & $2.1 \pm 2.9$ & $1.0(0.0-9.0)$ & \\
\hline \multirow{6}{*}{ Day 3} & Control & 10 & $0.3 \pm 0.5$ & $0.0(0.0-1.0)$ & \multirow{6}{*}{0.086} \\
\hline & PRF & 10 & $0.0 \pm 0.0$ & $0.0(0.0-0.0)$ & \\
\hline & Essix retainer & 10 & $0.0 \pm 0.0$ & $0.0(0.0-0.0)$ & \\
\hline & Ozone & 10 & $0.9 \pm 1.2$ & $0.0(0.0-3.0)$ & \\
\hline & LLLT & 10 & $1.1 \pm 1.9$ & $0.0(0.0-5.0)$ & \\
\hline & Collagen fleece & 10 & $0.7 \pm 1.5$ & $0.0(0.0-4.0)$ & \\
\hline \multirow{6}{*}{ Day 4} & Control & 10 & $2.4 \pm 2.8$ & $1.5(0.0-9.0)$ & \multirow{6}{*}{0.105} \\
\hline & PRF & 10 & $0.1 \pm 0.3$ & $0.0(0.0-1.0)$ & \\
\hline & Essix retainer & 10 & $1.0 \pm 2.2$ & $0.0(0.0-6.0)$ & \\
\hline & Ozone & 10 & $2.4 \pm 3.0$ & $0.5(0.0-7.0)$ & \\
\hline & LLLT & 10 & $1.9 \pm 2.8$ & $0.5(0.0-8.0)$ & \\
\hline & Collagen fleece & 10 & $2.4 \pm 3.1$ & $1.0(0.0-9.0)$ & \\
\hline \multirow{6}{*}{ Day 5} & Control & 10 & $3.9 \pm 3.1$ & $4.0(0.0-9.0)$ & \multirow{6}{*}{$0.011^{*}$} \\
\hline & PRF & 10 & $0.3 \pm 0.7$ & $0.0(0.0-2.0)$ & \\
\hline & Essix retainer & 10 & $1.1 \pm 2.1$ & $0.0(0.0-5.0)$ & \\
\hline & Ozone & 10 & $4.0 \pm 2.8$ & $4.0(0.0-9.0)$ & \\
\hline & LLLT & 10 & $1.5 \pm 2.6$ & $0.5(0.0-8.0)$ & \\
\hline & Collagen fleece & 10 & $2.8 \pm 3.8$ & $1.0(0.0-9.0)$ & \\
\hline \multirow{6}{*}{ Day 6} & Control & 10 & $2.4 \pm 3.2$ & $1.0(0.0-8.0)$ & \multirow{6}{*}{0.223} \\
\hline & PRF & 10 & $0.1 \pm 0.3$ & $0.0(0.0-1.0)$ & \\
\hline & Essix retainer & 10 & $1.1 \pm 2.1$ & $0.0(0.0-5.0)$ & \\
\hline & Ozone & 10 & $1.7 \pm 1.9$ & $1.0(0.0-5.0)$ & \\
\hline & LLLT & 10 & $1.1 \pm 2.8$ & $0.0(0.0-9.0)$ & \\
\hline & Collagen fleece & 10 & $1.6 \pm 2.9$ & $0.0(0.0-9.0)$ & \\
\hline \multirow{6}{*}{ Day 7} & Control & 10 & $1.9 \pm 2.0$ & $1.5(0.0-5.0)$ & \multirow{6}{*}{0.186} \\
\hline & PRF & 10 & $0.4 \pm 1.0$ & $0.0(0.0-3.0)$ & \\
\hline & Essix retainer & 10 & $0.6 \pm 1.6$ & $0.0(0.0-5.0)$ & \\
\hline & Ozone & 10 & $0.6 \pm 1.1$ & $0.0(0.0-3.0)$ & \\
\hline & LLLT & 10 & $0.6 \pm 1.6$ & $0.0(0.0-5.0)$ & \\
\hline & Collagen fleece & 10 & $1.6 \pm 2.2$ & $0.5(0.0-5.0)$ & \\
\hline
\end{tabular}




\section{DISCUSSION}

The definition of the quality of life is "a life without diseases from the perspective of patients". The purpose of this concept is to ensure that people achieve their objectives and choose an ideal lifestyle. A patient-based assessment of the condition of health is essential for measuring wellbeing. Recently, patient-based assessments are considered critical endpoints in assessment of treatment outcomes, and the need for such assessments has led to development of the methods for quality of life scales related to oral health. ${ }^{30}$ OHIP- $49^{31}$ and its shorter version OHIP- $14^{12}$ are the ones that are most comprehensive, accessible and most frequently used among those scales. OHIP measures the social impact of oral diseases on overall health as perceived by individuals, and it is used worldwide. Although it is a physiometric principle that reliability of an index decreases statistically in proportion to the number of questions, it is necessary for a pragmatic scale system to be easy and simple to implement. Therefore, in the present study, Turkish version of the OHIP-14 scale was used to identify the life quality of patients had FGG procedures with regard to post-operative palatal wound healing and their levels of perception of post-operative symptoms, and to compare the effects of different treatment methods on palatal wound area.

FGG is an approach that is considered gold standard with a proven success in treatment of mucogingival deformities and increasing the amount of attached gingiva. ${ }^{22}$ Since the palatal donor site is left for secondary healing, the bleeding, pain, edema and infection observed after the harvesting of FGG are among the complications that appear at the first week after the operation. $^{20}$ Various treatment approaches are utilized on the donor site in order to reduce bacterial contamination and accelerate wound healing so that such complications are minimized and better conditions of post-operative wound care are provided..$^{20}$

Recent studies have examined the postoperative morbidity of periodontal surgery and its effects on the quality of life. ${ }^{32,33} \mathrm{~A}$ recent study reported by McGuire et al. ${ }^{34}$ highlighted to raise awareness about the reliability, validity, sensitivity and clinical applicability of the use of current approaches for examination of post-operative morbidity and healing in periodontal surgery. Tonetti et $a l .{ }^{35}$ reported in their study which evaluated the post-operative healing period of periodontal plastic surgery using the scores obtained with OHIP-14 scale and showed a tendency to return to the patients' pre-operative scores at a 14-day follow-up period after the operation despite the initial increase.

The findings of our study showed that FGG operation had a negative effect on the patients' quality of life in the early postoperative healing period. In consisted with, Taşdemir et al. ${ }^{36}$ showed that harvesting deepithelialized gingival graft affected the patients' quality of life in the early healing period. That study indicated that a statistically significant increase was observed in the OHIP-14 scores on day 6 after the operation, compared to the baseline condition, and that this effect started to decrease at week 2 postoperatively. It was also stated that the test group showed a better quality of life compared to spontaneous healing on day 6 after the operation, in parallel with the VAS values. It was particularly consistent with our study findings that the control group (spontaneous healing) showed higher VAS values regarding postoperative pain on days 1,6 and 7 after the operation. These findings could be attributed to the fact that donor sites of the patients in the control group are more sensitive to stimulation since they are left for secondary healing.

The previous study reported by Özçelik et al..$^{37}$ which treated gingival recession using deepithelialized palatal graft, measured the patients' quality of life by OHQoL scale and showed that the values in the quality of life scale tended to return to the baseline values at week 1 after the operation. They pointed out in their study that VAS scores and OHQoL scale values of the test group were in correlation after the biostimulation of the operated area by using a diode laser and discussed that this difference may be attributed to the analgesic impact of laser therapy which accelerated the wound healing. In our study, the LLLT group had the lowest scores for the 
physical pain, psychological discomfort and physical disability sub-scales of the OHIP-14 on day 14 after the operation. Furthermore, it was found statistically significant that application of LLLT had a positive effect with regard to the matters of diet and embarrassment expressed by the question 7 and 10 of the OHIP-14 scale. It was observed that the patients in the collagen group were disturbed the most by the sub-scales of physical pain, physical disability, social disability and handicap. This result could be explained by the potential disturbance caused by the sutures that was used to immobilize the collagen fleece or the potential allergenic characteristics of collagen.

Femminella et al. $^{21}$ assessed the postoperative wound healing and morbidity by applying PRF and gelatin sponge on the palatal wound area following the harvesting of epithelialized FGG. It was reported in the study that PRF provided a significant clinical benefit in accelerating the healing of palatal wounds and reducing post-operative morbidity by stimulating angiogenesis and epithelialization by the cytokines, glycoproteins and growth factors that it contains. In parallel with the results, PRF group showed significantly successful outcomes particularly in terms of post-operative pain in the VAS scale, moreover, the PRF group had lower scores compared to other groups for the sub-scales of physical disability and handicap with regard to the sub-scales of the OHIP-14 questionnaire.

Oliveira et al. ${ }^{38}$ used the OHIP-14 questionnaire in their study to assess the impact of oral conditions on the quality of life. The ROC curve had a $82.7 \%$ accuracy in that study. They noted that OHIP-14 had a high sensitivity in indicating the impact of gingival recession and cervical dentin on the patients' quality of life, and reported that this was, above all, related to the conditions of physical pain, and physical and psychological disability which are defined as the subscales of OHIP-14. In our study, we believe that the fact that the outcomes related to the post-operative physical pain in the OHIP-14 TR scale are observed with lowest scores in the LLLT group in contrast with the results of the VAS scale may be associated with a decrease in cervical dentin sensitivity in connection with the amount of root coverage on the recipient site on day 14 in addition to the treatments procedures on the donor site. On the other hand, the fact that essix retainer group had the highest psychological discomfort score may be attributed to the obligation of the patients to use this retainer perpetually after the operation and the difficulty of the patients to tolerate the retainer. In addition, the fact that the control group had the highest mean of psychological disability values may be explained by leaving the wound for spontaneous healing without applying any treatment modalities on the donor site.

The previous studies which examined the wound healing of the palatal donor site after harvesting autogenous grafts, noted that the graft sizes might be related to post-operative morbidity. ${ }^{39,40}$ In this sense, we aimed to standardize the size of the FGG for treatment of the deformities related to a single tooth zone in an effort to eliminate the potential differences. It should be considered that the differences of data among different groups arise from the patient's experience, duration of treatment, patient's reaction to anxiety/discomfort/pain, healing period and compliance with post-operative recommendation both during the treatment and post-operative healing period. In addition, it should take into account that different periodontal surgery procedures may affect the quality of life in different ways depending on the types of wound healing, i.e. primary or secondary healing of wound.

One limitation of the present study is that the study included a small study population and that no pre-study power analysis was possible to perform. However, as may be approved by many clinicians, the requirement to monitor the patients in defined time points frequently is the major challenge to increasing the patient population, and it is difficult to ensure maintenance of patients during the study period. Another limitation of the present study may be the fact that the number of analgesics taken by our patients could not be standardized. We think that this may have affected both the VAS values and certain sub-scales of the OHIP-14 questionnaire. In addition, even if the OHIP-14 scale was used to assess the patients' quality of life after the operation, non-comparison with the pre- 
operative OHIP-14 scores can be considered another limitation of this study.

\section{CONCLUSIONS}

Within the limitations of the present study, it was observed that the application of LLLT on the palatal donor wound site following the FGG procedures may have positive effects on physical pain, psychological discomfort and physical disability with regard to the quality of life. It was also observed that PRF applications may have a favorable impact on the patient comfort with respect to the postoperative pain.

\section{ACKNOWLEDGEMENTS}

None

\section{CONFLICTS OF INTEREST}

None

\section{Serbest Dişeti Grefti Uygulanan Hastalartn Ağız Sağlığı ile İlişskili Yaşam Kalitesinin Değerlendirilmesi $\ddot{O} Z$}

Amaç: Ăğı săğlı̆g ile ilişkili yaşam kalitesi bireyin, ă̆ız sağllğının yaşam kalitesi ve genel sağlı̆ etkilediğini kişisel olarak algılamasıdır. Periodontal plastik cerrahi prosedürlerinin operasyon sonrast hastaların yaşam kalitesini negatif olarak etkilediği bildirilmişstir. Bu çalışmanın amacl, serbest dişeti grefti (SDG) uygulamasinı takiben verici bölgenin iyileşmesi için uygulanan farkl tedavi prosedürlerinin yaşam kalitesi üzerine etkisini değerlendirmektir. Gereç ve Yöntemler: SDG operasyonu sonrası, 60 palatal donör bölge, her bir grupta 10 hasta olacak şekilde, randomize olarak altı gruba ayrılmıştır. Palatal yara bölgeleri trombositten zengin fibrin (TZF), essix plağl, ozon tedavisi, düşük-doz lazer tedavisi (DDLT) veya kolajen sünger materyali uygulamalarından biri ile tedavi edilmiştir. Kontrol grubuna herhangi bir tedavi uygulanmayarak sekonder iyileşmeye birakulmuştır. Hastaların cerrahi işlem sonrast yaşam kalitesi Ăğz Sağlı̆̆l Etki Profili-14 (OHIP-14) ölçeği ve postoperatif rahatsızlıklarına iliş̧kin parametreler ise Görsel Analog Skalası (VAS) ile değerlendirilmiştir. Bulgular: OHIP14 ölçeğinin Türkçe versiyonuna (OHIP-14 TR) ilişkin sorularda, grup nitel değişkenleriyle soru 7 ve soru 10 nitel değişkenleri arasinda istatistiksel olarak anlaml iliş̧ki bulunmuştur ( $p=0,002$ ve $p=0,015)$. Bu sorulara ait en düşük skorlar en çok DDLT grubunda verilmistitir. Toplam OHIP skoru (TOHIP) ve OHIP-14 anketinin 7 alt ölçeğine ait değerleri gruplar arasında istatistiksel açıdan herhangi bir fark göstermemiştir $(p>0,05)$. VAS ölçeğine göre postoperatif ăgr düzeyleri değerlendirildiğinde, operasyon sonrast 5. günde TZF ile ozon grupları arasindaki fark istatistiksel olarak anlamlllk göstermiştir $(p=0,011)$. Sonuçlar: SDG operasyonunu takiben verici yara bölgesine uygulanan DDLT yönteminin yaşam kalitesinde olumlu etkiler oluşturabileceği ve TZF uygulamasinin operasyon sonrast hasta konforu açısından daha etkili olabileceği gözlenmiş̧tir. Anahtar Kelimeler: Hemostatik yöntemler, lazer tedavisi, oral cerrahi işlemleri, yaşam kalitesi.

\section{REFERENCES}

1. Koller M, Klinkhammer-Schalke M, Lorenz W. Outcome and quality of life in medicine: a conceptual framework to put quality of life research into practice. Urol Oncol 2005;23:186-192.

2. Hegarty AM, McGrath C, Hodgson TA, Porter SR. Patient-centred outcome measures in oral medicine: are they valid and reliable? Int J Oral Maxillofac Surg 2002;31:670-674.

3. Frisch MB, Hoboken NJ. Quality of Life Therapy: Applying a Life Satisfaction Approach to Positive Psychology and Cognitive Therapy. New Jersey: John Wiley \& Sons Inc, 2006: 47.

4. Slade GD, Spencer AJ. Development and evaluation of the Oral Health Impact Profile. Community Dent Health 1994;11:3-11.

5. Allen PF. Assessment of oral health related quality of life. Health Qual of Life Outcomes 2003;8:1-40.

6. Locker D, Allen F. What do measures of 'oral healthrelated quality of life' measure? Community Dent Oral Epidemiol 2005;35:401-411.

7. McGrath C, Bedi R. An evaluation of a new measure of oral health related quality of life-OHQoL-UK(W). Community Dent Health 2001;18:138-143.

8. Cunha-Cruz J, Hujoel PP, Kressin NR. Oral healthrelated quality of life of periodontal patients. J Periodontal Res 2007;42:169-176.

9. Jansson $\mathrm{H}$, Wahlin A, Johansson V, Akerman S, Lundegren N, Isberg PE, et al. Impact of periodontal disease experience on oral health-related quality of life. J Periodontol 2014;85:438-445.

10. Levin L, Zini A, Levine J, Weiss M, Lev RA, Hai A, Chebath-Taub D, Almoznino G. Dental anxiety and oral health-related quality of life in aggressive periodontitis patients. Clin Oral Investig 2018;22:1411-1422.

11. He S, Wei S, Wang J, Ji P. Chronic periodontitis and oral health-related quality of life in Chinese adults: A population-based, cross-sectional study. J Periodontol 2018;89:275-284.

12. Slade GD. Derivation and validation of a short-form oral health impact profile. Community Dent Oral Epidemiol 1997;25,284-290.

13. Marquez IC. The role of keratinized tissue and attached gingiva in maintaining periodontal/periimplant health. Gen Dent 2004;52:74-78.

14. Sanz M, Simion M, Working Group 3 of the European Workshop on Periodontology. Surgical techniques on periodontal plastic surgery and soft tissue regeneration: consensus report of Group 3 of the 10th European Workshop on Periodontology. J Clin Periodontol 2014;41(Suppl 15):92-97. 
15. Farnoush A. Techniques for the protection and coverage of the donor sites in free soft tissue grafts. J Periodontol 1978;49:403-405.

16. Burkhardt R, Hammerle $\mathrm{CH}$, Lang NP, Research Group on Oral Soft Tissue B, Wound H. Self-reported pain perception of patients after mucosal graft harvesting in the palatal area. J Clin Periodontol 2015;42:281-287.

17. Brasher WJ, Rees TD, Boyce WA. Complications of free grafts of masticatory mucosa. J Periodontol 1975;46:133-138.

18. Wang HL, Bunyaratavej $P$, Labadie M, ShyrY, MacNeil RL. Comparison of 2 clinical techniques for treatment of gingival recession. J Periodontol 2001;72: 1301-1311.

19. Patel PV, Kumar S, Vidya GD, Patel A,Holmes JC, Kumar V. Cytological assessment of healing palatal donor site wounds and grafted gingival wounds after application of ozonated oil: an eighteen-month randomized controlled clinical trial. Acta cytologica 2012;56:277-284.

20. Keceli HG, Aylikci BU, Koseoglu S, Dolgun A. Evaluation of palatal donor site haemostasis and wound healing after free gingival graft surgery. J Clin Periodontol 2015;42:582-589.

21. Femminella B, Iaconi MC, Di Tullio M, Romano L, Sinjari B, D'Arcangelo C, et al. Clinical Comparison of Platelet Rich Fibrin and a Gelatin Sponge in the Management of Palatal Wounds Following Epithelialized Free Gingival Graft Harvest: A Randomized Clinical Trial. J Periodontol 2015:1-17.

22. Wang CY, Tsai SC, Yu MC, Lin YF, Chen CC, Chang PC. $660 \mathrm{~nm}$ LED Light Irradiation Promotes the Healing of the Donor Wound of Free Gingival Graft. J Periodontol. 2015;86:674-681.

23. Thoma DS, Sancho-Puchades M, Ettlin DA, Hammerle CH, Jung RE. Impact of a collagen matrix on early healing, aesthetics and patient morbidity in oral mucosal wounds - a randomized study in humans. J Clin Periodontol 2012;39:157-165.

24. Sullivan HC, Atkins JH. Free autogenous gingival grafts. I. Principles of successful grafting. Periodontics 1968;6:121-129.

25. Miller PD Jr. A classification of marginal tissue recession. Int J Periodontics Restorative Dent 1985;5:8-13.

26. Dohan DM, Choukroun J, Diss A, Dohan SL, Dohan AJJ, Mouhyi J, et al. Platelet-rich fibrin (PRF): a secondgeneration platelet concentrate. Part I: technological concepts and evolution. Oral Surg Oral Med Oral Pathol Oral Radiol Endod 2006;101:37-44.

27. O'Leary TJ, Drake RB, Naylor JE. The plaque control record. J Periodontol 1972;43:38.

28. Löe H, Silness J. Periodontal disease in pregnancy I. Prevalence and severity. Acta Odontol Scand 1963;21:533-551.

29. Mumcu G, Inanc N, Ergun T, Ikiz K, Gunes M, Islek U, Yavuz S, Sur H, Atalay T, Direskeneli H. Oral health related quality of life is affected by disease activity in Behçet's disease. Oral Dis 2006; 12:145-151.

30. $\mathrm{Ng}$ SK, Leung WK. Oral health-related quality of life and periodontal status. Community Dent Oral Epidemiol 2006;34:114-122.

31. Slade GD, Spencer AJ. Development and evaluation of the oral health impact profile. Community Dent Health 1994;11:3-11.

32. Tan WC, Krishnaswamy G, Ong MM, Lang NP. Patient-reported outcome measures after routine periodontal and implant surgical procedures. J Clin Periodontol 2014;41:618-624.

33. Mei CC, Lee FY, Yeh HC. Assessment of pain perception following periodontal and implant surgeries. J Clin Periodontol 2016;43:1151-1159.

34. McGuire MK, Scheyer ET, Gwaltney C. Commentary: Incorporating patient-reported outcomes in periodontal clinical trials. J Periodontol 2014;85: 1313-1319.

35. Tonetti MS, Cortellini P, Pellegrini G, Nieri M, Bonaccini D, Allegri M, et al. Xenogenic collagen matrix or autologous connective tissue graft as adjunct to coronally advanced flaps for coverage of multiple adjacent gingival recession: Randomized trial assessing non-inferiority in root coverage and superiority in oral health-related quality of life. J Clin Periodontol 2018;45:78-88.

36. Taşdemir Z, Alkan BA, Albayrak H. Effects of ozone therapy on the early healing period of deepithelialized gingival grafts: A randomized placebocontrolled clinical trial. J Periodontol 2016;87:663-671.

37. Ozcelik O, Seydaoglu G, Haytac CM. Diode laser for harvesting de-epithelialized palatal graft in the treatment of gingival recession defects: a randomized clinical trial. J Clin Periodontol 2016;43:63-71.

38. Douglas de Oliveira DW, Marques DP, AguiarCantuária IC, Flecha OD, Gonçalves PF. Effect of surgical defect coverage on cervical dentin hypersensitivity and quality of life. J Periodontol 2013;84:768-775.

39.Zucchelli G, Mounssif I, Mazzotti C, Montebugnoli L, Sangiorgi M, Mele M, Stefanini M. Does the dimension of the graft influence patient morbidity and root coverage outcomes? A randomized controlled clinical trial. J Clin Periodontol 2014;41:708-716.

40. Burkhardt R, Hammerle CH, Lang NP, Research Group on Oral Soft Tissue Biology \& Wound Healing. Self-reported pain perception of patients after mucosal graft harvesting in the palatal area. J Clin Periodontol 2015;42:281-28 